\title{
Design Strategy of Information Construction Based on User Participation
}

\author{
Jundong Gao ${ }^{1, a}$ \\ 1Department of industrial design, Huazhong University of Science and Technology
}

\begin{abstract}
In the design of the Internet products, the information construction is one of the important factors to determine that whether a product is friendly. It is a challenge for every information architect to discover new innovations on the basis of established user habits. In practice, we often found that the development of many new functions did not meet the needs of users and many functions would be improved by users themselves, the fact of which shows the strong desire of users to participate in the development of products. Therefore, this article proposes an information architecture method based on the "users' partially involved", that is, when the main framework of a product is determined and the detailed architecture of part of the functions is proposed by users themselves, builders will help users to dig and organize their requirements and eventually integrate them into the design of architecture. In order to verify the effectiveness of this method, the article takes the virtual products for social service based on "surrounding geographic information" as an example. A comparison experiment was conducted on the effectiveness of the design of user-participant information architecture, and the experimental results were evaluated.
\end{abstract}

\section{Literature Review}

The architecture of information is a task that designers, developers, and content decision makers often need to face. As the bones of product, we should try to keep it simple and clear, rather than being easily changed, making users overwhelming[1]. Therefore, the information architecture of the product, which is of great significance to the product itself and the users' experience, needs a scientific and logical way to combine.

Scholars began to research the methodology of information architecture in 1970s, long before the emergence of the website and smart phone App. It originated from the methodology which is still used by many UX designers nowadays, and has three main categories, Library Information Science, Cognitive Psychology and Architecture[2]. The Bibliography and Archives of Library Information Science provide the organization method for information architecture.

In the research of design category of information architecture, An organization called UXbooth divides the content of information architecture into seven categories: User Research and Analysis, Create Navigation and Hierarchy, Framework, Tag, Taxonomy and Metadata, Data Modeling Parallel Literature[3], In the previous architecture design, information architects often construct the content architecture diagram and the wireframes with the procedure according to the data analysis, and classify the function level of the sub information in the wireframe, share the data with team in the form of prototype, pictures or suggestions, so that we can show the typical users of products, the logic and interaction path of functional requirements, and the way to use App.

But in this architecture approach, the degree of user involvement in product design is shallow, the form tends to be passive, Users mainly accept interviews, accept observation, fill in questionnaire and do usability test of the product according to the task set by the researcher, most of them make passive choices from different schemes, and express their views. Whether it is in the user's subjective feelings, or in the designers' and researchers' cognition, the users are objects of study, are cooperative party[4]. In

${ }^{a}$ Corresponding author: gaojunku2008@163.com

(C) The Authors, published by EDP Sciences. This is an open access article distributed under the terms of the Creative Commons Attribution License 4.0 (http://creativecommons.org/licenses/by/4.0/). 
recent years, with the form of users involved in the design of is used in the new design strategy, the roles of designer and user have subtly changed in comparison with tradition: the user becomes the product designer and changer, while designers and development engineers are more like coordinators and observers in PD session, perceptually getting the users' first-hand information. In this process, Researchers can tap users' consciousness and demand from a deeper perspective as the main organizers of the PD. Compared with the traditional design pattern, PD extremely emphasize taking the initiative and enthusiasm of the user who is no longer just passive from different schemes to make choice, to express their views, but really participates in the prototype design, designs in the way of brainstorm, and even be absorbed into the design team, working with designers in a short period of time. With the help of the materials provided by the experimenter, the user can provide the design proposal and explain his creative ideas. In the whole process, the user feels that he is working with the designer to create and solve the problem, he is the changer of the product.

This paper will take the mobile application of smart phone as the research object, to explore the design patterns in which the users will also partly participate, present a method of information architecture of mobile product, and verify the effectiveness of this method through the A/B test.

\section{Pre-study - research purposes and research object analysis}

The aim of this study is to test the effectiveness of information architecture design method with partial participation of user, Information architecture design method in the past, the APP information architecture is often through direct extraction of competing goods architecture, and then combined with the function design of the product itself, this method is based on the Angle of product architecture to think. And the proposed approach is obtained by using the method of user part of information architecture, he is a need to combine product and user mental models it is concluded that the method of architecture itself. The study object is the APP based on geographic location service, and the main function of such APP is to help people making online food ordering and seat reservation and arrange offline dining accordingly, to save user's time of waiting for empty seat and queuing, so that help people solve their dinning demand in restaurant through queuing. on one hand, it was such a behavior developed from actual living behavior to the internet then to mobile internet, with the typical significance; on the other hand, the reason is that, for such behavior of online food booking and offline dining, the user may have quite large difference in the original intention of product's developers, for product development, the developers may consider more about product itself, but the user may consider that the software usage scenario and using habit shall have the same important significance with the experience of product itself.

\section{Experiment I - the information architecture design based on competing product thought}

The aim of this study is to use general method to abstract the information architecture of product A, the abstract method is usually the methods provided by product's decision-maker, without the protruded content irrelevant to core function, it can enable the user completing the task and fulfill the demand of product itself accordingly.

First of all, we selected to sort the logic of original product, made information architecture analysis and task process analysis, to perform segmentation firstly according to the application's functional module, to abstract the architecture of dinning part and refine it in single function, further to draw all the functions in the tree structure. From such a task process we can find out user's core using function, then to finally result in the architecture based on product thinking by combining the core functions and according to the structure of competing product, and manufacture the prototype A accordingly. 

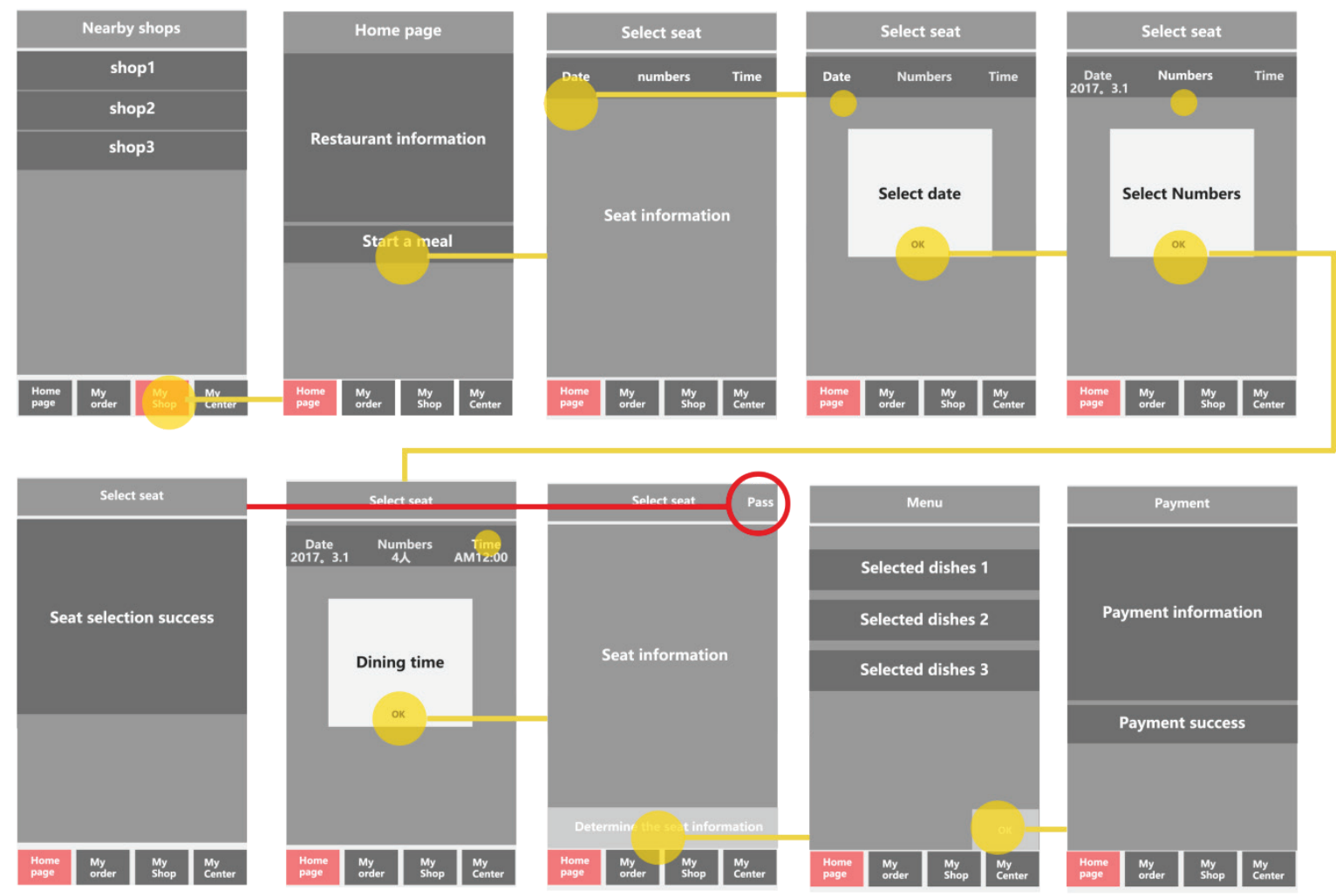

Figure 1.Test prototype A

\section{Experiment II - the information architecture strategy design based on partial participation of users.}

This study is the focus of the whole experiment, in order to introduce the information architecture approach for the part of the user.

The first step is to find three products based on geographic location as the competing products to analyze the object of analysis. They are respectively the three mainstream geographic information service applications as Meituan.com, Alipay's public praise and Dianping.com. Unlike previous methods, the aim of our competing product analysis is to analyze the generality and difference among the architectures, in other words, it refers to make better innovation with the premise that do not impede user's habit. On one hand, to analyze the already formed habits of the user in the part of generality, for example, if the navigation in certain level and logic of core function in many competing products both used the same or similar processing method, this means that such part of architecture or user's using habit was already stable, without the enough assurance, it cannot make the blind innovation for representing the difference, otherwise it may bring the huge study cost for the user and influence the usage experience of the product. On the other hand, to find out the innovative point of the architecture in the differences.

Through the analysis on the competing products, we have found the generality of the three architectures: the first one, The user's mental model and usage logic have been relatively stable in the organization architecture of selecting location information and time information, and in the user core usage process, users' mental model and using logic was relatively stable, the user has also formed the solid habit in the two using methods of seat selection and food booking, during the architecture sorting these cannot be changed easily. The second, the overall layout of the information architecture in the three competing products are basically the same, especially the core usage process based on food booking, for the content layout in home page, they both used the label navigation, so we've also used the same organization method to control experiment effect. The third, the organizational architecture of competing products did control the vertical level depth in 4 stories, but had quite little differences in horizontal range, "Alipay" and Ele.me maintained the four dimensions range, but "Meituan" gave up the "discover" bar, maintained in three dimension range. From the perspective of cognitive psychology, we'd control user's vertical and horizontal cognitive load within 4 stories approximately. 


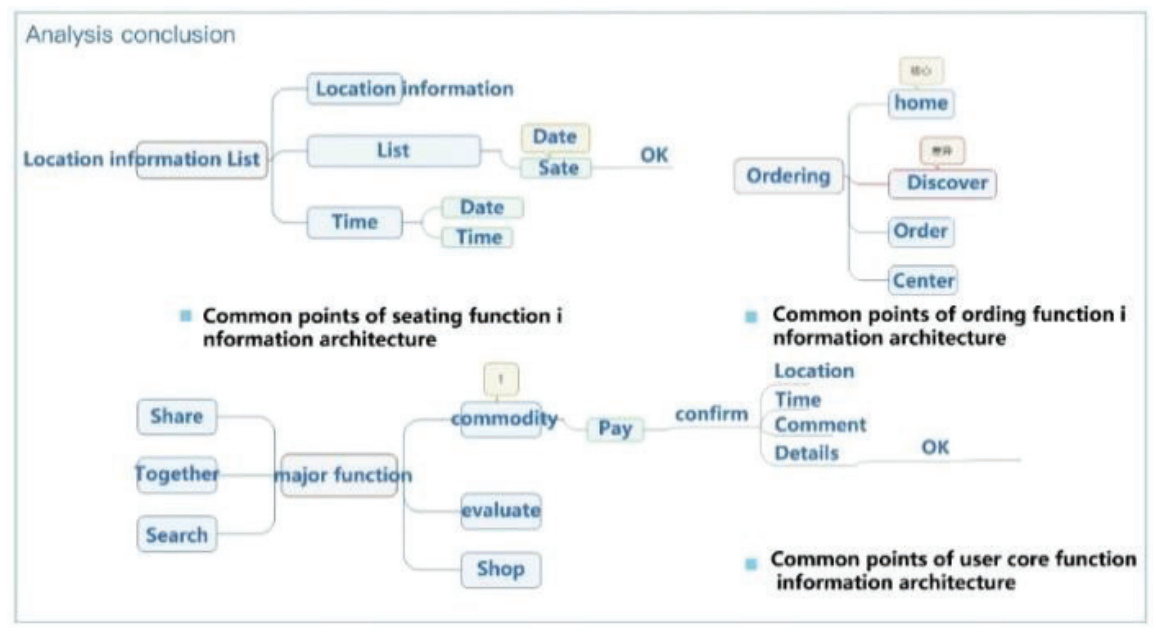

Figure 2. Similarities and differences in architecture

The second step, we've used card classification method to collect the information architecture based on user mental model.

First of all, we handled the target users with disorganized end information through architecture A, the designer's task was to interpret the function of each card, ensuring that the users have had completely understand the function of these cards, then the participants were organized and classified by themselves, and also arrange the name for each category. The process of guiding user to classify the information into different grades may need 2 or 3 times, so as to ensure the user classification being not too concentrated or dispersed. Then the relative functional cards were classified according to their importance degree, during this process the record was also maintained very well, finally it can result in the classification based on user mental model, by comparing the difference in the competing products and user mental model classification to record them, finally to organize the organizational structure based on user mental model. At last, to disorganize the functional elements of relative web pages at the place with such difference, let the users make the combination within the range provided by us, the aim is to sort out the related function of important level and find out the problems and needs, We have come to some conclusion in the experiment that users often classify order and reservations as one category, After an interview analysis, we decided to classify the booking and ordering as a class and maintain them at the same level .In the primary navigation, Product decision makers hope keeping "my shop" to facilitate the promotion of products, But this classification makes the user confused in the process of using the product, After the interview found that the user needs is to choose from a number of stores to enter, so the choice of business and determine the target business is a complete logic, you should not split their classification, so in the back of the structure we simplify one Level navigation for three categories and finally result in the architecture with partial participation of users, and make the prototype B accordingly.

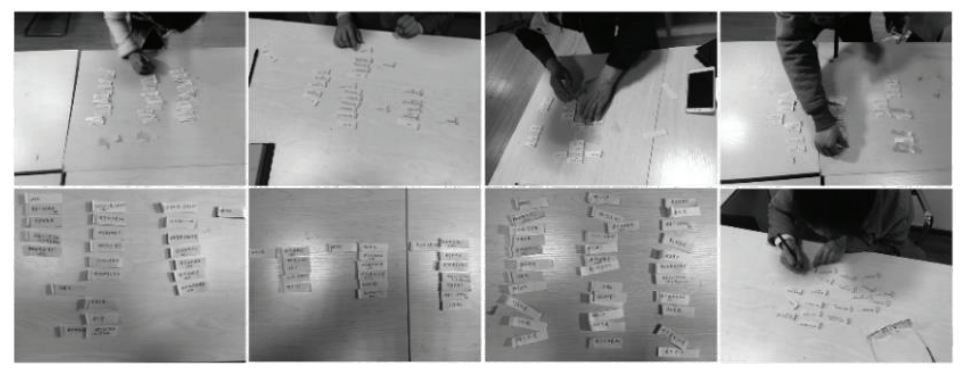

Figure 3.User participation in design process 


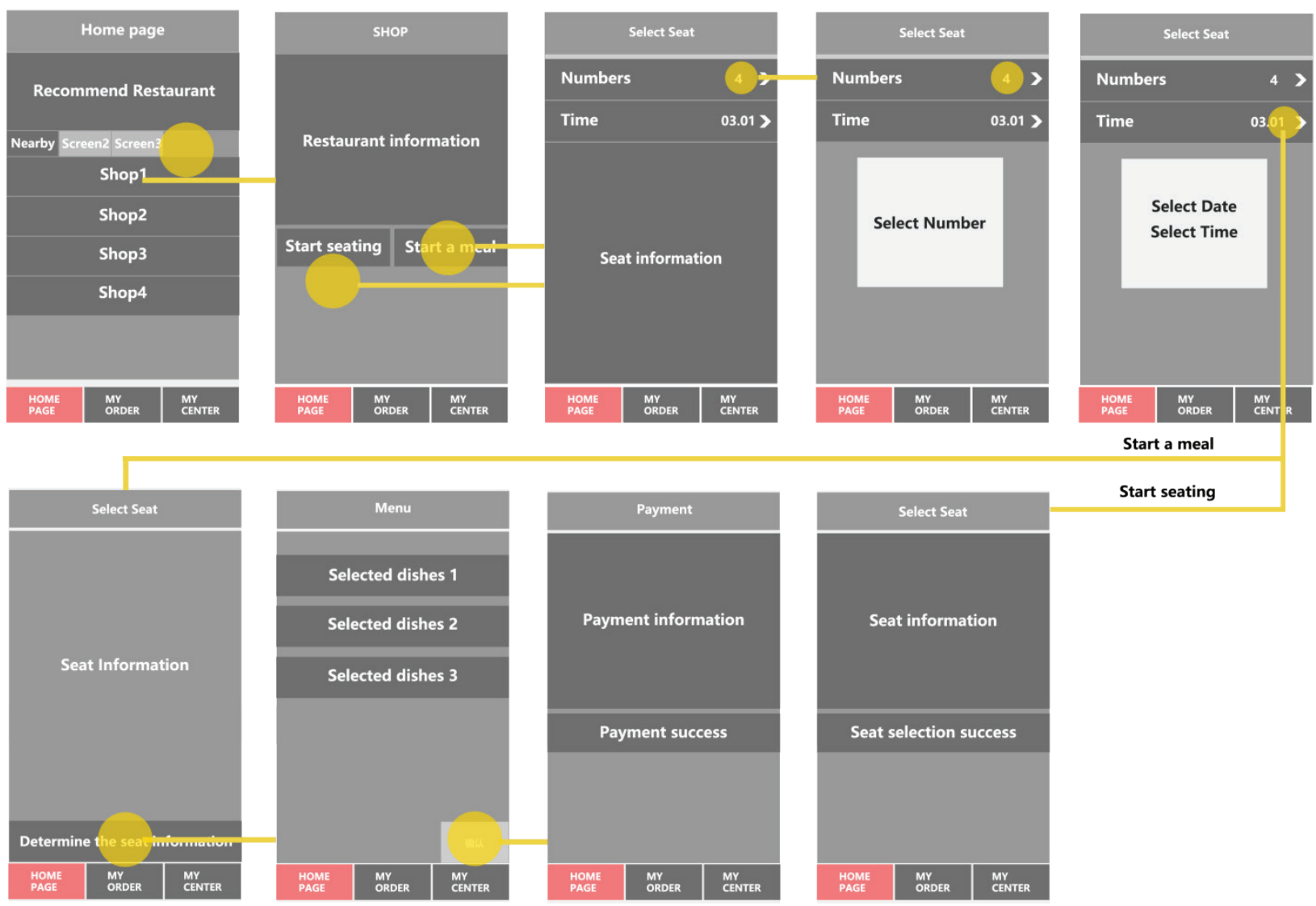

Figure 4.Test prototype B

\section{Usability testing and result analysis}

The main aim of this study is to discuss the effectiveness of design strategy with partial participation of users, the study methods include the objective performance of prototype A and B (product usability test) and subjective feeling (USE questionnaire)

The independent variable in this experiment can be divided in four task categories, they are respectively the completion of seat booking, completion of food booking, selecting restaurant nearby, find the completed order, the control variables are the equipment platform, visual factor. Based on the independent and control variables, we've used the product usability test and USE questionnaire to collect the performance measurement of participants completing the set task, the main dependent variables include the task successful degree, completion time of the task, completion efficiency, error rate and effectiveness, usability, learnability and satisfactory degree.

The participants in this experiment were the 15 college students whose age varied between 18 to 25 , it included 8 females and 7 males, 9 of them were studied in the major of industrial design, other 6 came from the majors of mechanics, materials respectively. All the participants are the user who often used APP in daily life.

From the overall level it can be seen that: 1) there's difference in user experience comprehensive assessment of prototype A and B in 4 tasks, prototype B's comprehensive score was higher than that of A, the reason may be that the prototype B based on user mental model can even better match the using habits of the users. 2) in task 1, prototype A's effectiveness and usability were significantly better than prototype A, the reason may be that in prototype A the information level of the function "seat reservation" was intentionally weakened in product's consideration dimension, the user may pay more attention on food booking but ignore the functional position of seat reservation. 3 ) in task 2, the comprehensive score of the two prototypes are almost the same, the reason may be that it both considered user's core usage process in prototype A and B. 


\begin{tabular}{|r|r|r|r|r|r|r|}
\hline & learning-ease & using-ease & Satisfaction & Effectiveness & Overal1 merit \\
\hline 1 & 4.39 & 3.53 & 4.47 & 3.91 & 4.075 \\
\hline 2 & 3.65 & 4.11 & 3.63 & 4.62 & 4.0025 \\
\hline 3 & 4.15 & 3.72 & 3.3 & 3.55 & 3.68 \\
\hline 4 & 3.38 & 4 & 4.12 & 4.29 & 3.9475 \\
\hline 5 & 3.62 & 3.38 & 3.79 & 4.02 & 3.7025 \\
\hline 6 & 4.03 & 4.19 & 4.2 & 4.39 & 4.2025 \\
\hline Average & 3.87 & 3.82166667 & 3.918333333 & 4.13 & 3.935 \\
\hline Median & 3.84 & 3.86 & 3.955 & 4.155 & 3.975 \\
Geometric me & 3.854392756 & 3.80970806 & 3.898692364 & 4.114961964 & 3.930416884 \\
standard dev & 0.380893686 & 0.32896302 & 0.425742489 & 0.382151802 & 0.2073403 \\
\hline
\end{tabular}

Table 1. Test results of prototype A

\begin{tabular}{|r|r|r|r|r|r|r|}
\hline & learning-ease & using-ease & Satisfaction & Effectiveness & Overal1 merit \\
\hline 1 & 4.56 & 4.79 & 4.18 & 4.32 & 4.4625 \\
\hline 2 & 4.09 & 4.3 & 4.68 & 3.6 & 4.1675 \\
\hline 3 & 4.21 & 3.89 & 4.11 & 3.93 & 4.035 \\
\hline 4 & 4.18 & 4.53 & 3.72 & 4.68 & 4.2775 \\
\hline 5 & 3.95 & 4.38 & 4.05 & 4.32 & 4.175 \\
\hline 6 & 4.14 & 4.1 & 3.6 & 4.27 & 4.0275 \\
\hline Average & 4.188333333 & 4.33166667 & 4.056666667 & 4.186666667 & 4.190833333 \\
\hline Median & 4.16 & 4.34 & 4.08 & 4.295 & 4.17125 \\
\hline Geometric me & 4.184311367 & 4.32200193 & 4.041979161 & 4.172433069 & 4.188224027 \\
\hline standard dev & 0.203707306 & 0.31669649 & 0.381715426 & 0.373077293 & 0.163138489 \\
\hline
\end{tabular}

Table 2. Test results of prototype B

\section{References}

1. Norman, D A, The Design of Everyday Things[M]. New York: Doubleday, 1990

2. Lindgard G, Usability Testing and System Evaluation: A Guide for Designing Useful Computer Systems, Chapman and Hall, London, U.K, 1994

3. Tian Lei, Shuaili Wei. An Exploration of Relationships between Culture Images and User Experience of Gesture Interaction. In: Lasse Berntzen, Vestfold University College eds. CENTRIC 2013. Venice. 2013.pp 112-117.

4. Liu, Y.C., Ma, Y.H.: The Behavior Analysis of Product Negative Word-of-Mouth Spread on Sina Weibo. Human Centered Computing, pp. 284-295(2016) 